\title{
COMPARATIVE EVALUATION OF FLUORIDE VARNISH SINGLE AND RE APPLICATION ON DEMINERALIZATION ADJACENT TO BONDED BRACKETS
}

\author{
Wadih Abou Hamdan * and Sherine Badr **
}

\begin{abstract}
Aim: was to compare the effectiveness of single application of fluoride vanish ${ }^{\mathrm{TM}}$ (3M Unitek, Monrovia, CA, USA) to its reapplication in preventing enamel demineralization around orthodontic brackets, using a laser fluorescence monitoring device on a group of human extracted teeth in vitro.

Methods: Orthodontic brackets were bonded to 64 extracted premolars. Teeth were divided into two groups: group A and group B, each of 32 teeth. Each group was subjected to two demineralization cycles in standard demineralization solution. For group A fluoride vanish ${ }^{\mathrm{TM}}$ (3M Unitek, Monrovia, CA, USA) was applied around the orthodontic brackets only once before the 1 st demineralization cycle, while for group B fluoride vanish ${ }^{\mathrm{TM}}$ was applied twice before both the 1st and 2nd demineralization cycle. Enamel demineralization was measured for both groups at baseline, after first demineralization cycle, and after second demineralization cycle using laser fluorescence device Diagnodent pen. Data were analyzed using Friedman test followed by Wilcoxon test. Differences were considered statistically significant at $\mathrm{p}<0.00$.
\end{abstract}

Results: Statistically significant differences were observed between the two groups after the second demineralization cycle $\mathrm{p}<0.001$. Lesser demineralization was found in group B where fluoride varnish was applied twice.

Conclusion: Fluoride varnish reapplication is more effective in preventing enamel demineralization around orthodontic brackets than single application.

KEYWORDS: Fluoride varnish reapplication, Demineralization, Diagnodent pen.

\section{INTRODUCTION}

Enamel demineralization involves the loss of calcified tooth structure leading to an altered surface look, known as White spot lesions (WSLs).
WSLs can be defined as porosity in subsurface of demineralized enamel that appears as a milky white localized opacity on smooth surfaces (Bishara SE and Ostby AW 2008).

* BDS, Faculty of Dentistry, Beirut Arab University, Lebanon

** BDS, MSc, PhD, DPHE, Associate Professor of Pediatric Dentistry, Faculty of Dentistry, Beirut Arab University,

Lebanon, Faculty of Oral and Dental Medicine, Cairo University, Egypt. 
This problem is aggravated in orthodontic patients. Throughout orthodontic treatment, demineralization of the enamel around brackets is a frequent incidence with poor oral hygiene patients. Fixed appliance makes oral hygiene very difficult, it creates an environment that favors the onset of enamel demineralization and dental plaque formation and restricts the cleaning effect of saliva (Derks et al., 2007). Previous studies have shown that enamel demineralization could develop in orthodontic patients in just four weeks (Boersma JG et al., 2005). Moreover, the presence of unattractive WSLs may contradict the objective of orthodontic treatment to improve dental and facial esthetics which might be distressing to both patients and orthodontists.

Orthodontists have tried to diminish demineralization with limited success. As an example, dentifrices and fluoride solutions were successfully used to reduce demineralization but patients don't use these materials regularly. Geiger noticed that only $47.5 \%$ of the patients used fluoride solutions daily at home (Geiger AM et al., 1992).

Preventive measures that require no patient compliance would seem to be more convenient for the orthodontic patients. Ogaard B et al 1997 observed a reduction of enamel demineralization by $48 \%$ after application of fluoride varnish. More recent studies, Vivaldi Rodrigues showed that fluoride varnish applications every three months after start of treatment reduced the incidence of WSLs by 44\% (Vivaldi R et al., 2006).

Not only, fluoride varnish application is a preventive method that requires no patient cooperation, but also it is affordable, easy to use and most importantly, gives the orthodontist the ability to control the amount and frequency of fluoride application, and can be easily adapted to orthodontic bonding protocols (Bowman, S.J 2000). Moreover fluoride varnishes provide high concentration of fluoride as they can adhere to enamel surface longer than other fluoride applications that_require patient regular use (Zabokova E et al., 2012).
Vanish $^{\mathrm{TM}}$ (3M Unitek, Monrovia, CA, USA) is a viscous resinous lacquer consisting of 5\% sodium fluoride white varnish with tri-calcium phosphate (TCP) that hardens into a white coating. Fluoride ions can be combined into the hydroxyl-apatite structure of enamel by the replacement of hydroxyl groups forming less soluble fluoridated forms, such as fluorhydroxyl-apatite (Chang M.C. 2008). Also, varnishes containing TCP have an advantage of superior protection against lesion initiation and progression and enhancing enamel remineralization (Patil N et al., 2013 and Tosun S et al., 2016).

Diagnodent pen has been used widely both in vivo and in vitro for detecting enamel demineralization (Vivaldi R 2006 and Sichani A et al., 2016). This portable hand held instrument also uses fluorescence laser to distinguish between the demineralized and healthy tooth structure and measures laser fluorescence within tooth structure. When the laser light is spread into the site, two-way hand-piece optics allows the device to concurrently measure the reflected laser light energy (Mepparambath $\mathrm{R}$ et al., 2014).

Topical fluoride agents had a great value in decreasing enamel demineralization both invitro and in-vivo studies (Mdos $\mathrm{N}$ et al., 2016 and Da Camara D et al., 2016). However, limited studies tested the efficiency of fluoride varnish reapplication in preventing white spot lesion formation in orthodontic patients (Baygin $\mathrm{O}$ et al., 2013, Nalbantgil D et al., 2013, and Restrepo M et al., 2016). Thus this study was conducted to investigate if reapplication of fluoride varnish would have superior benefit on single application in preventing enamel demineralization around orthodontic brackets.

The Aim of this study was to compare the effectiveness of single application of fluoride vanish $^{\text {TM }}$ to its reapplication in preventing enamel demineralization around orthodontic brackets, using a laser fluorescence monitoring device on a group of human extracted teeth in vitro. 


\section{MATERIAL AND METHODS}

Study design: A comparative controlled in-vitro study.

Study setting: The research project was conducted at Beirut Arab University, Lebanon.

Study sample: The study was conducted on a sample of 64 sound premolars extracted for orthodontics purposes from patients with age range between 15 and 25 years old. Simple visual examination by magnifier $(\times 5)$ and sharp pointed explorer was done to exclude any teeth with carious, fracture, stains, restoration, fluorosis, and any clinical evidence of demineralization. Teeth were cleaned from blood and soft tissue. After debridement, teeth were stored in saline $(0.9 \%$ $\mathrm{NaCl}$ ) solution. Metal stainless steel 0,18 slot Roth system (3M Unitek, Monrovia, CA, USA ) brackets were bonded using a light cured composite resin and adhesive (Reliance West Thorndale Ave Itasca United States) in accordance with the manufacturer's instructions.

The sixty four teeth were divided into two groups: group A and group B, each of 32 teeth. For group A fluoride varnish was applied around the orthodontic brackets only once before the $1^{\text {st }}$ demineralization cycle, while for group B fluoride varnish was applied twice before both the $1^{\text {st }}$ and $2^{\text {nd }}$ demineralization cycle.

Baseline measurement (M1) of the enamel on the buccal surface of each tooth was assessed immediately after bonding using a laser fluorescence device (Diagnodent pen KaVo, Biberach an der Riss, Germany) (Figure 1). This device uses laser with 655 -nanometer diode to identify smooth surface and non-cavitated demineralization before they are noticeable by naked eye or radiograph. The tip of the device measures the reflected laser fluorescence from tooth surface indicating the degree of demineralization. Higher readings indicate greater demineralization. Readings runs from 0 to 99 , the scales found in normal patient are: $0-10=$ sound tooth enamel; 11-20 =outer demineralized half enamel; 21-30 = inner demineralized half enamel;

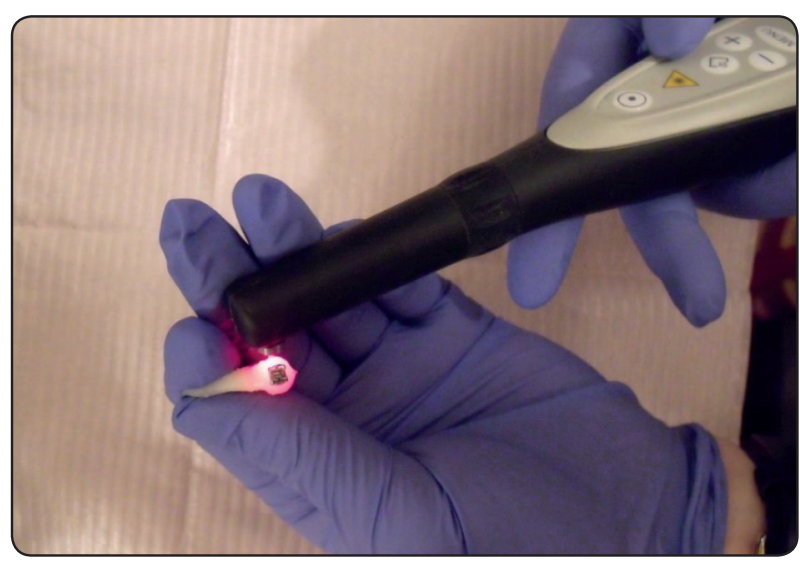

Fig. (1) Measurement after bonding using Diagnodent-pen.

$30+=$ dentinal caries (Matheus $\mathrm{P}$ et al., 2010). For teeth to be included in this study baseline measurements should range from 0 to 10 .

After baseline measurement, fluoride varnish $5 \%$ sodium fluoride (white varnish) with tri-calcium phosphate Vanish $^{\text {TM }}$ (3M Unitek, Monrovia, CA, USA) was applied on the buccal surface with thin micro-brush for both groups.

All samples were placed in standard demineralizing solution of $\mathrm{pH}=4.4(2.20 \mathrm{mM} \mathrm{Ca}+2$, $2.20 \mathrm{mM} \mathrm{PO} 43,0.05 \mathrm{M}$ acetic acid and $0.025 \mathrm{ppm} \mathrm{F}$ ) for four days to create demineralized lesions (White DJ 1995), the solution was changed every 6 hours (Figure 2). These four days of demineralization cycle represents approximately 3 months of real time in the oral cavity (Sudjalim TR et al 2007).

After the first demineralization cycle all teeth were cleaned with water and dried, then the second measurement (M2) was registered. Only for group B fluoride varnish was reapplied. Then all samples of group A and group B were immersed again in demineralization solution for an additional four days. After the second demineralization cycle, the third measurements (M3) were registered.

\section{Statistical analysis}

Statistical analysis was performed using SPSS for Windows version 18.0. The alpha error was set at 0.05 . 


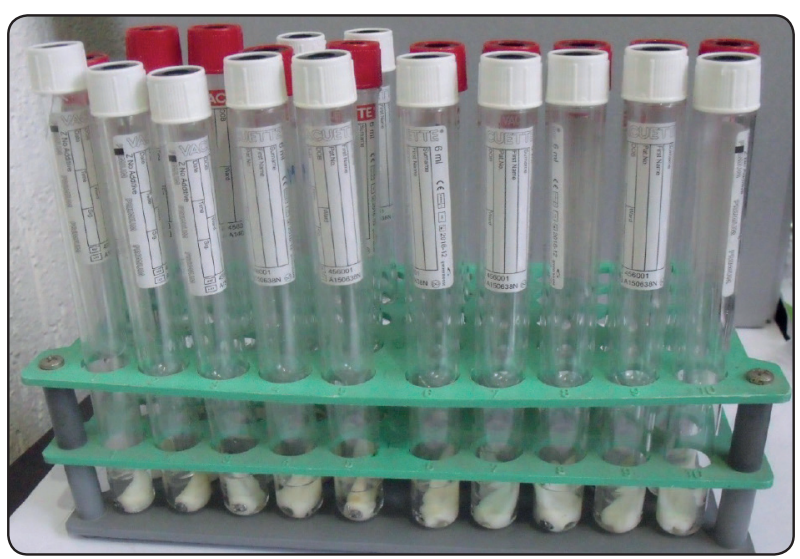

Fig. (2) Samples placed in standard demineralizing solution

Data were presented as mean and standard deviation (SD) of the degree of enamel demineralization. Kolmogorov-Smirnov tests were carried out to assess the normality distribution of continuous variables in different groups. Since these variables were not normally distributed, non-parametric tests were used for statistical comparisons.

Friedman tests followed by Wilcoxon tests (after alpha error correction) were performed to compare the degree of demineralization over a period of time (at Baseline (M1), after the first demineralization cycle (M2), and after the second demineralization cycle (M3)) for both groups A and $\mathrm{B}$.
The sample power was calculated using the G-Power 3.1.3 power analysis software. The minimum required samples for Friedman test followed by Wilcoxon test, with alpha of 0.05 , is 20 samples in each group. Instead, in this study, 32 samples were used in each group in order to achieve the highest possible reliability.

\section{RESULTS}

Table 1 shows the mean degree and standard deviation of demineralization over time at baseline (M1), after the first demineralization cycle (M2), and after the second demineralization cycle (M3) for groups A and B.

The mean degree and standard deviation $(\mathrm{M} \pm \mathrm{SD})$ of demineralization at baseline (M1), after the first demineralization cycle (M2), and after the second demineralization cycle (M3) for group A were $5.91 \pm 1.174,8.63 \pm 1.040$ and $11.38 \pm 1.212$ respectively, while for group B were $6.04 \pm 1.160$, $8.10 \pm 1.101$ and $9.90 \pm 1.160$ respectively. There was a statistically significant difference between the mean degrees of demineralization over a period of time for both groups A and $\mathrm{B}(\mathrm{P}<0.001)$.

At baseline (M1) there was no significant difference between group A and group B ( $>>0.05)$ where the mean degrees and standard deviation of demineralization for groups $\mathrm{A}$ and $\mathrm{B}$ were

TABLE (1) Mean degree and standard deviation of demineralization over a period of time for group A and group B

\begin{tabular}{|l|c|c|c|c|}
\hline $\begin{array}{r}\text { Mean Degree of } \\
\text { demineralization }\end{array}$ & Baseline (M1) & $\begin{array}{c}\text { After first cycle of } \\
\text { demineralization (M2) }\end{array}$ & $\begin{array}{c}\text { After second cycle of } \\
\text { demineralization (M3) }\end{array}$ & P-value \\
\hline group A (N=32) & $5.91 \pm 1.174$ & $8.63 \pm 1.040$ & $11.38 \pm 1.212$ & $<0.001$; Friedman test \\
\hline group B (N=32) & $6.04 \pm 1.160$ & $8.10 \pm 1.101$ & $* 9.90 \pm 1.160$ & $<0.001$; Friedman test \\
\hline P-value & $>0.05$;Wilcoxon test & $>0.05$; Wilcoxon test & $<0.001$; Wilcoxon test & \\
\hline
\end{tabular}

* Where fluoride varnish has been reapplied 


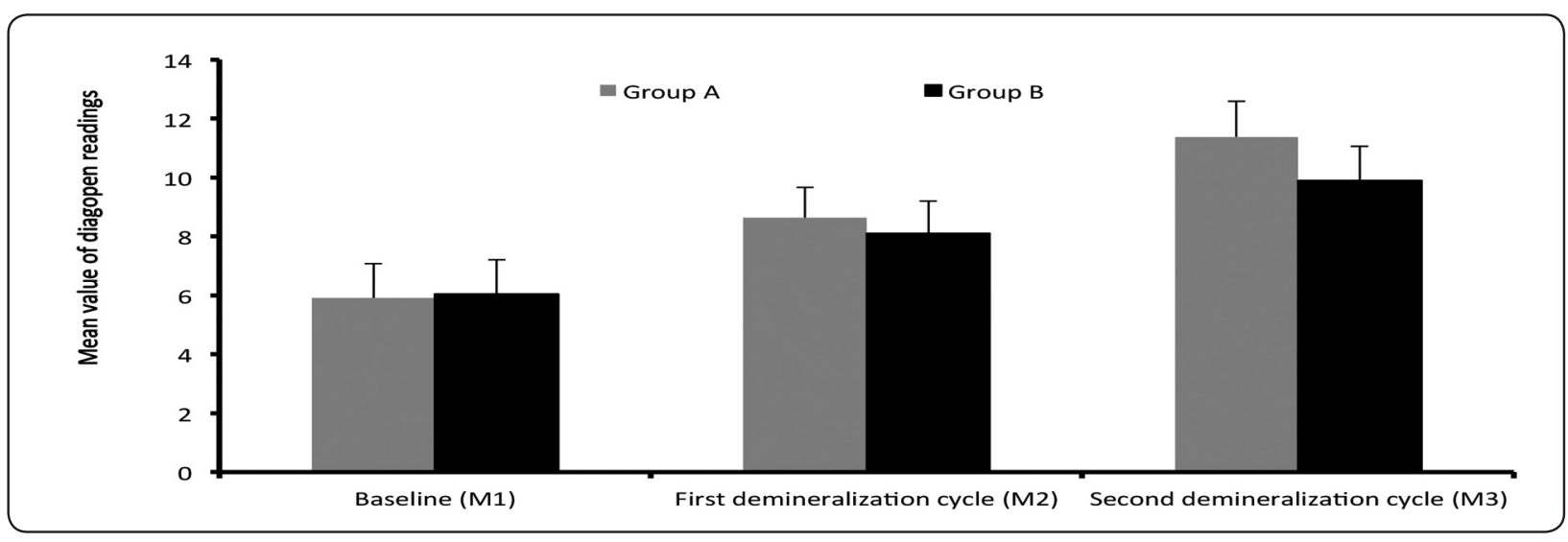

Fig. (3) Mean values of diagnodent pen readings for Groups A and B

$(5.91 \pm 1.174$ and $6.04 \pm 1.160$ respectively). Similarly, after the first cycle of demineralization (M2) the mean degree and standard deviation of demineralization for groups $\mathrm{A}$ and $\mathrm{B}$ was $(8.63 \pm 1.040$ and $8.10 \pm 1.101$ respectively) with no statistical significant difference between them.

After the second demineralization cycle the mean degrees and standard deviation of demineralization for group A and group B were $(11.38 \pm 1.212$ and $9.90 \pm 1.160$ respectively) with a statistically significant difference at $\mathrm{P}<0.001$, where the mean degree of demineralization has decreased significantly in group B where fluoride varnish was applied twice compared to group A where fluoride varnish was applied only once (Table 1) (figure 3).

\section{DISCUSSION}

Throughout orthodontic treatment, orthodontic brackets and bands promote more plaque retention and make oral hygiene difficult. Fluoride varnishes could be used as a preventive method. Fluoride varnish application around bonded brackets has been proven to decrease the incidence of WSLs. Studies have shown that fluoride varnish with $5 \%$ sodium fluoride reduce WSLs incidence by about 50\% (Schmit IL 2002 and Carina D 2011). Even though it was noticed that fluoride varnishes cannot totally inhibit white spots lesion formation (Schmit IL 2002). Therefore, periodic application of fluoride can provide an effective clinical solution. Thus, the aim of the study was to compare in-vitro the effect of a single fluoride varnish application to its reapplication in preventing enamel demineralization around orthodontic brackets.

Sixty four freshly extracted premolars were divided into 2 groups each of 32 teeth. In the first group fluoride was applied once before the first demineralization cycle, while in the second group fluoride varnish was applied twice before each demineralization cycles. Both groups were subjected to immersion in two demineralization cycles. Each cycle was for four days in demineralization solution in order to develop demineralized lesions, representing approximately 3 months of real time effect (Sudjalim TR et al 2007).

Assessments of the degree of demineralization were done at baseline, after each demineralization cycle using laser fluorescence device (Diagnodent pen). Baseline assessment was done to determine the readings of the samples before starting the experiment and to make sure that there is no statistical difference between readings of teeth in both groups. Readings were measured after each demineralization cycle to show the effect of fluoride varnish on demineralization and compare it to baseline readings and to compare readings of both groups. 
Diagnodent pen was used in this study to detect enamel demineralization as it is currently coincided to be one of the most accurate devices for detecting enamel demineralization and it has been used widely both in-vivo and in-vitro (Vivaldi R 2006, Mepparambath R et al., 2014 and Sichani A et al., 2016). With respect to the diagnosis of dental caries, the conventional methods (visual and radiographic examination) present low sensitivity for quantifying the changes in mineral content resulting from demineralization.

Vanish $^{\text {TM }}$ was used in this study because it is cheaper, easier to apply, and requires no patient compliance in comparison to other fluoride products like fluoride rinses, gels and dentifrice. Unlike conventional varnishes, Vanish ${ }^{\mathrm{TM}}$ contains modified rosin that is white or tooth-colored, so after its application on the teeth the product is virtually invisible. Also, this product contains tri-calcium phosphate (TCP) which is considered the main agent for enamel remineralization. When TCP interacts with demineralized enamel it starts to remineralize the lesion. Karlinsey in 2009 found that TCP fluoride varnish had better efficacy in inhibiting progression of initial primary enamel lesions than Duraphat $\AA($ Karlinsey RL et al., 2009). The positive effect of fluoride varnish showed in this study was supported with other reports (Bowman S.J 2000, Zabokova E et al., 2012 and Carina D, 2011). Two of these investigators used Duraflor varnish that contains $5 \%$ of sodium fluoride as Vanish ${ }^{\mathrm{TM}}$.

The results of this study show that the mean degree of enamel demineralization in group B where fluoride varnish was applied twice before each demineralization cycle was significantly lower than that of group A, where fluoride was applied only once. Therefore, fluoride varnish reapplication decreased the mean degree of demineralization.

The positive effect of fluoride varnish reapplication was also supported by other study (Virupaxi S et al., 2016) in which they found that the reapplication of fluoride varnish decreased the demineralization and prevent or control white spot lesion progression. Moreover, Restrepo $\mathrm{M}$ et al., 2016 found the same positive effect of fluoride varnish reapplication till the fourth application only, further applications didn't show any positive effect (Restrepo M et al., 2016). This is due to the fact that fluoride varnish flows and adheres to the teeth surface and as it comes in contact with saliva, it slowly wears away over time, and the sodium fluoride and calcium phosphate in the varnish dissolve and are released as ions. Consequently, fluoride ions react with free calcium, and along with the available calcium ions they combine to form insoluble calcium fluoride which aids in enamel protection (Chang M.C 2008). Calcium phosphate salts, such as tri-calcium phosphate (TCP) added to the varnish will improve the enamel remineralization of white spot lesions (Karlinsey RL et al., 2009).

\section{CONCLUSIONS}

From the results of this study and within its limitation it can be concluded that fluoride varnish reapplication is more effective in preventing enamel demineralization around orthodontic brackets than single application.

\section{Limitations}

The assessment of enamel demineralization can be done using more than one detection technique in order to have more accurate comparable readings such as polarized light microscopy, microradiographs, and/or micro-hardness testing.

\section{RECOMMENDATIONS}

Based on the clinical findings of this study, orthodontists should be aware of the great value of fluoride varnish application and its reapplication every two to three months during orthodontic treatment. Furthermore, using fluoride varnishes is an ideal choice for reducing enamel decalcification around orthodontic brackets. 


\section{REFERENCES}

1. Baygin O, Tuzuner T, Ozel M.B, Bostanoglu O. (2013). Comparison of combined application treatment with onevisit varnish treatments in an orthodontic population. Med Oral Patol Oral Cir Bucal. ; 18:62-70.

2. Bishara SE, Ostby AW.(2008) White spot lesions: Formation, prevention, and treatment. Seminars in Orthodontics; 14:174-82.

3. Bowman S.J (2000). Use of a fluoride varnish to reduce decalcification, J. Clin. Orthod. ;34:377-379.

4. Boersma JG, van der Veen MH, Lagerweij MD, Bokhout B (2005).Caries prevalence measured with QLF after treatment with fixed orthodontic appliances: influencing factors. Caries Res.; 39:41-7.

5. Carina D, Gustavo D. Andilson R (2011). Efficacy of a fluoride varnish in preventing white spot lesions as measured with laser fluorescence. J Clin Orthod. ;45:25-9.

6. Chang M.C. (2008). Fluoride incorporation in hydroxyapatite. Journal of Materials Science: Materials in Medicine; 19:2837-43.

7. Derks A, Kuijpers A.M, Frencken J.E, Van't Hof M.A ,Katsaros C (2007). Caries preventive measures used in orthodontic practices: An evidence-based decision? Am. J. Orthod. ; 132:165-170.

8. Da Camara D, Pessan J, Francati T, Souza J, Danelon M, Delbem A. (2016).Fluoride toothpaste supplemented with sodium hexametaphosphate reduces enamel demineralization in vitro. Clinical Oral Investigations; 20:1981-1985.

9. Geiger A.M, Gorelick L, Gwinnett A.J, Benson B.J(1992) .Reducing white spot lesions in orthodontic populations with fluoride rinsing, American Journal of Orthodontics and Dentofacial Orthopedics;101:403-407.

10. Karlinsey RL, Mackey AC, Stookey GK, Pfarrer AM (2009). In vitro assessments of experimental NaF dentifrices containing a prospective calcium phosphate technology. Am J Dent.; 22:180-4.

11. Matheus P, Demito C.F, Scheibel P.C, Bowman S.J, Ramos A.L (2010).Correlation between the microscopic evaluation and reading by laser fluorescence of enamel decalcification in bovine teeth: In vitro study, Odonto. ; 18:31-39.

12. Mepparambath R, Bhat S, Hegde S, Anjana G, Sunil M, Mathew S(2014). Comparison of proximal Caries detection in primary teeth between laser fluorescence and Bitewing radiography: An in vivo study. Int $\mathrm{J}$ Clin Pediatr Dent.;:163-7.

13. Mdos N, Romão D, Cury J, Tabchoury, C. (2016) .Effect of fluoride concentration on reduction of enamel deminer- alization according to the Cariogenic challenge. Brazilian dental journal; 27:393-8.

14. Nalbantgil D, Oztoprak M, Cakan D, Bozkurt K,Arun T (2013) .Prevention of demineralization around orthodontic brackets using two different fluoride varnishes. European journal of dentistry; 7:41-47.

15. Ogaard B, Duschner H, Ruben I, Arends (1997). Microradiography and confocat laser scanning microscopy applied to enamel lesions formed in vivo with and without fluoride varnish treatment. Eur J OralSci: 104:378-83.

16. Patil N, Choudhari S, Kulkarni S,Joshi S. (2013).Comparative evaluation of remineralizing potential of three agents on artificially demineralized human enamel: An in vitro study. J. Conserv. Dent. ; 16:116-20.

17. Restrepo M, Bussaneli D, Jeremias F, Cordeiro R, Raveli D, Magalhães A., Candolo C, Santos-Pinto L. (2016). Control of white spot lesions with use of fluoride varnish or Chlorhexidine gel during orthodontic treatment A Randomized clinical trial. The Journal of clinical pediatric dentistry; 40:274-80.

18. Sudjalim TR, Woods MG, Manton DJ, Reynolds EC (2007). Prevention of demineralization around orthodontic brackets: in vitro. Am J Orthod Dentofac.; 131:705-9.

19. Sichani A, Javadinejad S, Ghafari R (2016) .Diagnostic value of DIAGNOdent in detecting caries under composite restorations of primary molars. Dent.Res.J.;13:327-332.

20. Schmit IL, Staley RN, Wefel S, Kanellis M. Jakobsen JR(2002). Keenan PI. Effect of fluoride varnish on demineralization adjacent to bonded brackets .Am J Orthod Dentofacial Orthop:122:125-134.

21. Tosun S, Culha E, Aydin U, Ozsevik A. (2016) .The combined occluding effect of sodium fluoride varnish and nd: YAG laser irradiation on dentinal tubules-a CLSM and SEM study. Scanning :38:619-624.

22. Vivaldi R, Demito CF, Bowman SJ, Ramos AL (2006). The effectiveness of a fluoride varnish in preventing the development of white spot lesions. World J. Orthod. ; 7:138-144.

23. Virupaxi S, Roshan N, Poornima P, Nagaveni N, Neena I, Bharath K. (2016).Comparative evaluation of longevity of fluoride release from three different fluoride varnishes - an In vitro study. Journal of clinical and diagnostic research; 10:33-6.

24. White DJ (1995). The application of in vitro models to research on demineralization and remineralization of the teeth. Adv Dent Res. ; 9:175-93.

25. Zabokova E, Sotirovska A, Evrosimovska B, Kanurkova L.(2012) Effect of Fluoride Varnish on Demineralization Adjacent to Orthodontic Brackets; Balk J Stom.; 16:157-160. 